\title{
„Entrepreneurial Orientation“: Eine Bestandsaufnahme der Konstruktschärfe
}
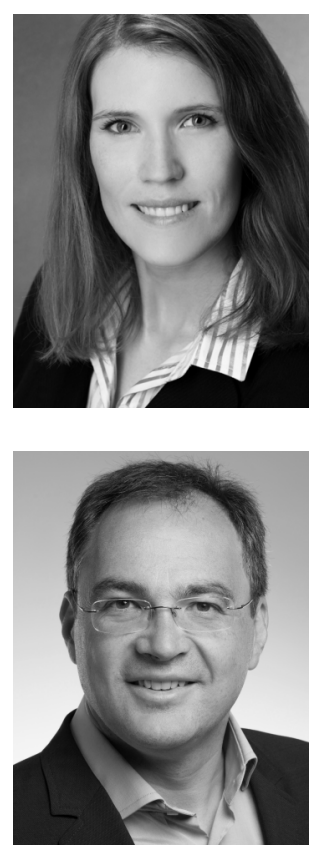

Simone A. Schweiger und Artur Baldauf ${ }^{1}$

Entrepreneurship, Entrepreneurial Orientation, Konstruktschärfe Entrepreneurship, Entrepreneurial Orientation, Construct Clarity

Entrepreneurial Orientation (EO) ist ein zentrales Konstrukt in der Management- und Entrepreneurship-Forschung. Trotz der häufigen Bezugnahme auf das Konstrukt im wissenschaftlichen und praktischen Diskurs werden grundlegende Debatten zu dessen Konzeptualisierung und Operationalisierung geführt. Diese Diskussionen betreffen die Dimensionalität des EO-Konstrukts, die Eignung der konzeptualisierten Dimensionen, die Operationalisierung durch eine Mischung aus einstellungs- und verhaltensbasierten Indikatoren und die Konzeptualisierung als reflektives oder formatives Konstrukt. Mit dem Ziel insbesondere bei Forschern ein besseres Verständnis für eine EO zu erreichen, haben wir anhand der Kriterien der Konstruktschärfe Empfehlungen für die zukünftige Anwendung des EOKonstrukts abgeleitet. Diese Empfehlungen leisten einen Beitrag zur EO-Forschung, indem wir eine Grundlage für eine einheitliche Konzeptualisierung des Konstrukts bereitstellen.

Entrepreneurial orientation (EO) has become a focal construct within management and entrepreneurship research. Despite the construct's popularity, debates have emerged concerning its construct clarity. In general, these debates refer to the construct's conceptualization and operationalization comprising issues such as the construct's dimensionality, the appropriateness of its dimensions, its operationalization with a mixture of attitudinal and behavioral items, and the conceptualization of $E O$ as a reflective or formative construct. With the aim to support researchers to gain a better understanding of $E O$, we developed recommendations throughout our review based on the criteria for construct clarity. These recommendations contribute to future research on $E O$ by providing a basis for a consistent conceptualization.

\section{Einleitung}

Im aktuellen wirtschaftlichen Umfeld erachten viele Organisationen unternehmerisches Agieren als eine zentrale Bedingung zur Erlangung von Wettbewerbsvorteilen. In der Management- bzw. Entrepreneurship-Forschung sind in der Vergangenheit auch umfassende Erkenntnisse generiert worden, welche zu einem besseren Verständnis der unternehmeri-

1 Wir danken Herrn Prof. Dr. Phillip Sieger und den anonymen Gutachtern für wertvolle Anregungen sowie Frau Julia Isensee für die Unterstützung bei der redaktionellen Erstellung der Arbeit.

Die Unternehmung, 70. Jg., 4/2016, DOI: 10.5771/0042-059X-2016-4-423 
schen Ausrichtung von Organisationen führten. Die Ergebnisse einer Metaanalyse (Rauch et al. 2009) dokumentieren die Bedeutung des Konstrukts „Entrepreneurial Orientation“ (EO) („unternehmerische Orientierung“) für den Unternehmenserfolg. Obwohl unterschiedliche Definitionen des EO-Konstrukts existieren, wird eine EO verstanden als „a firm's decision-making practices, managerial philosophies, and strategic behaviors that are entrepreneurial in nature " (Anderson et al. 2015). EO wird vor allem als ein auf Unternehmensebene bestehendes Phänomen angesehen, das eine unternehmerische Orientierung impliziert (z. B. Covin/Wales 2012) und die Kerndimensionen Innovativität, Proaktivität und Risikobereitschaft (Covin/Slevin 1989) reflektiert.

Erste akademisch fundierte Grundlagen in der EO-Forschung wurden vor allem von Mintzberg (1973), Khandwalla (1977) und Miller (1983) geschaffen, deren Beiträge als Pionierleistungen in der Management- bzw. Entrepreneurship-Forschung gelten. Mit Bezug auf diese fundamentalen Arbeiten, wurden durch Covin und Slevin (1989) sowie Lumpkin und Dess (1996) weitere Meilensteine im Erkenntnisgegenstand erreicht.

Intensives Forschungsinteresse entwickelte sich vor allem in Bezug auf die systematische Analyse des Einflusses einer EO auf den unternehmerischen Erfolg. Aufgrund inkonsistenter empirischer Ergebnisse (Brown et al. 2001; Covin et al. 1994; Kaya/Seyrek 2005; Rauch et al. 2009) ist insbesondere die mangelnde Konstruktschärfe von EO Gegenstand des wissenschaftlichen Diskurses. Hierbei werden die Dimensionalität des EO-Konstrukts, die Eignung der Dimensionen (z. B. Lumpkin/Dess 1996), die Operationalisierung mittels einstellungsund verhaltensbasierter Indikatoren (z. B. Miller 2011; Wiklund 1999) und die Konzeptualisierung als reflektiv oder formativ (z. B. George 2011) kontrovers diskutiert. Autoren fordern daher eine den wissenschaftlichen Regeln der Konstruktkonzeptualisierung und -operationalisierung entsprechende Weiterentwicklung von EO (z. B. Brown et al. 2001; Lumpkin et al. 2009; Lumpkin/Dess 2001; Rauch et al. 2009; Zahra et al. 1999).

Eine möglichst hohe Konstruktschärfe ermöglicht eine präzisere Kommunikation innerhalb einer Forschungsgemeinschaft (Podsakoff et al. 2016, Suddaby 2010). Dem EO-Konstrukt fehlt diese Schärfe, und klare Vorstellungen über die Definition sowie die Konzeptualisierung würden den theoretischen Fortschritt begünstigen (vgl. Lumpkin et al. 2009, 64; Zahra et al. 1999). In diesem Beitrag wollen wir demnach folgende Frage adressieren: «Wie können wir den Unklarheiten des EO-Konstrukts begegnen, um dessen Konstruktschärfe zu erhöhen?»

Im Folgenden diskutieren wir die Kriterien der Konstruktschärfe am Beispiel von EO und gehen dabei auf zentrale unter EO-Forschenden artikulierte Kritiken ein. Dabei integrieren wir das in der bestehenden Literatur zu wissenschaftlichen Konstrukten bestehende Wissen und leiten für jede identifizierte Unklarheit des Konstrukts Empfehlungen ab, um eine verbesserte Konstruktschärfe zu erlangen. Diese Empfehlungen leisten einen Beitrag zur EO-Forschung, indem wir eine Grundlage für eine einheitliche Konzeptualisierung des Konstrukts bereitstellen.

\section{Das Konstrukt „Entrepreneurial Orientation“}

In einer wegweisenden Studie haben Miller und Friesen (1978) elf Indikatoren zur Abbildung eines Strategie-Konstrukts - strategic posture - identifiziert. Miller (1983) hat vorgeschlagen, dass die Indikatoren zu Innovativität, Proaktivität und Risikobereitschaft die EO eines Unternehmens erfassen. Innovativität beschreibt «the disposition to engage in creativity and experimentation through the introduction of new products / services as well as 
technological leadership» (vgl. Rauch et al. 2009, 763). Proaktivität bezieht sich auf die Tendenz eines Unternehmens, zukünftige Nachfrage und Wettbewerb zu antizipieren und neue Produkte als Erstes auf den Markt zu bringen (Lumpkin/Dess 1996). Risikobereitschaft spiegelt die Tendenz eines Unternehmens wider, risikobehaftete Entscheidungen zu treffen, wie in unbekannten Geschäftsfeldern wettbewerbsaktiv zu werden oder hohe Kredite aufzunehmen (Lumpkin/Dess 1996; Rauch et al. 2009). Basierend auf Millers (1983) vorgeschlagenen Indikatoren haben Covin und Slevin (1989) die EO-Skala entwickelt, die jeweils drei Indikatoren für die Dimensionen Innovativität, Proaktivität und Risikobereitschaft enthält.

Seit der Einführung des EO-Konstrukts in der Managementliteratur im Jahr 1989 haben sich die wissenschaftlichen Ansprüche Konstruktkonzeptualisierung und -operationalisierung erhöht. Verfolgt man die EO-Konstruktmessung, so wird ersichtlich, dass die in aktuellen EO-Studien verwendeten Skalen häufig nicht der ursprünglichen Konzeption entsprechen. Obwohl sich die meisten Studien auf die Konstruktoperationalisierung von Covin und Slevin (1989) beziehen, wurde deren Skala zunehmend angepasst, um die Validität (z.B. interne Konsistenz oder inhaltliche Homogenität) zu verbessern. Ausserdem integrieren Forschende «items that discriminate at the desired level of attribute intensity» (vgl. Finn/Kayande 2004, 38). Skalenanpassungen finden vor der Datenerhebung statt und betreffen die Umformulierung, das Hinzufügen und / oder das Entfernen von Indikatoren sowie die Änderung des Skalenformats (z. B. semantische Differenz versus Likert-Skalen oder Skalen mit fünf versus sieben Punkten), während Skalenweiterentwicklungen auf psychometrischen Beurteilungskriterien basieren, wie das Hinzufügen und / oder Entfernen von Indikatoren zur unternehmerischen Orientierung nach der Datenerhebung (Finn/ Kayande, 2004).

Die EO-Skala nach Covin und Slevin (1989) wird im überwiegenden Teil der empirischen Studien vor der Datenerhebung angepasst. Indikatoren wurden insbesondere aufgrund kontextbezogener Spezifitäten der jeweiligen Studie hinzugefügt, entfernt und neu formuliert. Um eine adäquate Konstrukt-Reliabilität zu erreichen, wird die Skala häufig nach der Datenerhebung abgeändert. Forschende verwenden demnach zwar oftmals Covin und Slevins (1989) EO-Skala, verändern diese jedoch in der Mehrzahl der Studien. Die Vorgehensweise der Skalenanpassung begrenzt jedoch "our ability to compare and generalize research findings» (vgl. Hitt et al. 2004, 24). Diese Skalenanpassungen beruhen auf Kontroversen hinsichtlich verschiedener Aspekte des Konstrukts wie Dimensionalität, Definitionsbereich, Reliabilität sowie Validität. Trotzdem wird EO als ein zentrales und auch zukünftig notwendiges Konstrukt in der Entrepreneurship-Forschung erachtet (Covin) Lumpkin 2011). Im Einklang mit dieser Prämisse erscheint ein Konsens hinsichtlich der Konstruktdefinition sinnvoller als ein neues Konstrukt zu entwickeln, das potentiell besser, aber EO doch ähnlich ist (vgl. Hitt et al. 2004). Das erhebliche angesammelte Wissen würde ansonsten hinfällig, obwohl die bestehenden Unklarheiten des Konstrukts bewältigbar sind. Im Folgenden stellen wir demnach einen umfassenden Überblick über die Konzeptualisierung des Konstrukts dar, wobei wir auf die wesentlichen Diskussionspunkte rund um EO eingehen.

\section{Entrepreneurial Orientation und Konstruktschärfe}

Nach Suddaby (2010) wird die Konstruktschärfe anhand von vier Kriterien beurteilt: die Bereitstellung zufriedenstellender Definitionen, die Bestimmung des Geltungsbereichs des 
Konstrukts, die Darstellung der Beziehung zu anderen Konstrukten sowie das Aufzeigen von Kohärenz. Diese vier Beurteilungskriterien der Konstruktschärfe legen wir der Evaluation des EO-Konstrukts zugrunde.

In Bezug auf das Kriterium "zufriedenstellende Definiton" müssen Forschende grundlegende Eigenschaften und Charakteristika des Konzepts erläutern, Tautologien vermeiden und auf „Parsimonie ${ }^{2 " ~ a c h t e n . ~ F u ̈ r ~ d a s ~ K r i t e r i u m ~ „ B e s c h r e i b u n g ~ d e s ~ G e l t u n g s b e r e i c h s “ ~ i s t ~}$ es wichtig, dass Forschende den Kontext, in dem das Konstrukt sinnvoll angewendet werden kann, genau illustrieren. Dies ist von wesentlicher Bedeutung, da die meisten Konstrukte nicht universell sondern kontextspezifisch angewendet werden. Konstrukte unterliegen logischen Beziehungen zu anderen Konstrukten. Das Kriterium „Darstellung der logischen Beziehungen zu anderen Konstrukten“ erfordert das Einbetten eines Konstrukts in dessen nomologisches Netzwerk. Dies festigt das Verständnis für seinen konzeptionellen Definitionsbereich. Das Kriterium „Aufzeigen von Kohärenz“ bezieht sich auf die Frage, ob die grundlegenden Elemente des Konstrukts eine logische Konsistenz aufweisen. Dabei ist es wichtig, dass die Dimensionen logisch und empirisch überzeugend miteinander verbunden sind und dabei das latente Konstrukt erklären.

\subsection{Definition von Entrepreneurial Orientation}

In Tabelle 1 sind häufig verwendete EO-Definitionen dargestellt. Wie diese wenigen heterogenen Beispiele verdeutlichen, besteht Unklarheit bezüglich der dimensionalen Ausprägungen von Innovativität, Proaktivität und Risikobereitschaft sowie verhaltens- und einstellungsbasierter Indikatoren. Neben der Debatte hinsichtlich verhaltens- und einstellungsbasierter Indikatoren wird auch diskutiert, ob die ursprünglichen Dimensionen Innovativität, Proaktivität und Risikobereitschaft eine unternehmerische Orientierung adäquat repräsentieren.

\section{Tabelle 1: Beispiele für EO-Definitionen}

Autor(en) Definition von EO

Miller (1983: 771)

"An entrepreneurial firm is one that engages in product-market innovation, undertakes somewhat risky ventures, and is first to come up with "proactive" innovations, beating competitors to the punch."

Covin und Slevin (1989: 79)

EO "is characterized by frequent and extensive technological and product innovation, an aggressive competitive orientation, and a strong risktaking propensity by top management."

Lumpkin und Dess (1996: 136)

EO "refers to the processes, practices, and decision-making activities that lead to new entry."

Rauch et al. (2009: 762)

EO "refers to the strategy-making processes that provide organizations with a basis for entrepreneurial decisions and actions."

2 Unter Parsimonie wird in diesem Zusammenhang verstanden, dass eine Konstruktdefinition so knapp und präzise wie möglich die grundlegenden Eigenschaften eines Phänomens erfasst. Dabei soll die Definition die Bedeutung des Phänomens so eng wie möglich beschreiben, andererseits genügend Spielraum lassen um eine gewisse Generalisierbarkeit und praktikable Anwendbarkeit zuzulassen (Suddaby 2010). 


\subsubsection{EO als Einstellung versus Verhalten}

Miller $(1983,771)$ argumentierte, dass ein Unternehmen mit ausgeprägter EO eines ist, "that engages in product-market innovation, undertakes somewhat risky ventures, and is first to come up with "proactive» innovations, beating competitors to the punch». Basierend auf dieser Definition betrachteten Autoren EO aus einer Verhaltensperspektive (z. B. Green et al. 2008; Rauch et al. 2009; Zahra/Neubaum 1998). Alternativ wird EO auch als Einstellungsperspektive erachtet (z. B. Merz et al. 1994; Wiklund/Shepherd 2003), wobei sich Autoren häufig sowohl auf verhaltens- als auch einstellungsbasierte Aspekte beziehen, wenn sie EO definieren (z. B. Covin et al. 2006; Covin/Slevin 1989).

Aus verhaltensbasierter Perspektive werden Unternehmen, die eine EO besitzen, innovative, proaktive und risikoreiche Tätigkeiten zugeschrieben. Autoren, die diese Perspektive einnehmen, beziehen sich auf die Definition von Lumpkin und Dess (1996), wonach EO «the processes, practices, and decision-making activities that lead to new entry» umfasst (136). Diese «activities that lead to new entry» entsprechen dabei dem unternehmerischen Verhalten und enthalten beispielsweise "resource recombinations and entry into new markets or market segments» (vgl. Brown et al. 2001, 964).

In Bezug auf die einstellungsbasierte Perspektive von EO umschreiben Autoren das Konstrukt als eine Managementphilosophie (z. B. Anderson et al. 2009; Covin et al. 1990; Javalgi et al. 2012), eine strategische Haltung (Anderson et al. 2009; Covin et al. 1990; Dess et al. 1997; Heavey et al. 2009), eine Disposition (Matsuno et al. 2002), eine Präferenz (Covin et al. 2006; Matsuno et al. 2002), eine Neigung (z. B. Becherer/Maurer 1997; Matsuno et al. 2002; Morris/Paul 1987; Sapienza/Grimm 1997; Simsek et al. 2010; Walter et al. 2006) oder als eine Einstellung, sich mit unternehmerischem Verhalten zu beschäftigen (Naman/Slevin 1993; Wiklund 1998, 1999; Wiklund/Shepherd 2005).

Wenn sowohl verhaltens- als auch einstellungsbasierte Aspekte berücksichtigt werden, wird insbesondere die Skala von Covin und Slevin (1989) verwendet. Tatsächlich schlagen Covin und Slevin (1989) selbst eine Operationalisierung vor, die "a mix of current attitudes and past behaviors» darstellt (vgl. Brown et al. 2001, 954). Dies führte zu Verwirrung unter den Forschenden (Miller 2011). Tabelle 3 zeigt diese weitgehend genutzte EO-Skala von Covin und Slevin (1989), die jeweils drei Indikatoren für die Dimensionen der Innovativität (INN1, INN2 und INN3), Proaktivität (PRO1, PRO2 und PRO3) und Risikobereitschaft (RT1, RT2 und RT3) enthält. Die Indikatoren INN2, INN3, PRO1 und PRO2 sind auf momentane und vergangene Verhaltensweisen bezogen, während alle anderen Indikatoren aktuelle Einstellungen betreffen. 
Tabelle 2: EO-Skala von Covin und Slevin (1989)

In general, the top managers of my firms favor...

INN1 a strong emphasis on the marketing of tried and true products and services.
1 to 7

a strong emphasis on $\mathrm{R} \& \mathrm{D}$, technological leadership, and innovations.

How many new lines of products or services has your firm marketed in the past five years?

INN2 No new lines of products or services.

INN3

Changes in product or service lines have been mostly of a minor nature.
1 to $7 \begin{aligned} & \text { Very many new lines of products or } \\ & \text { services. }\end{aligned}$

1 to 7 Changes in product or service lines have usually been quite dramatic.

In dealing with competitors, my firm...

PRO1 typically responds to actions which competitors initate.

is very seldom the first business to introduce

PRO2 new products/services, administrative

techniques, operating technologies, etc.

PRO3 typically seeks to avoid competitive clashes, preferring a live-and-let-live posture.
1 to 7

typically initiates actions which competitors then respond to.

is very often the first business to introduce

1 to 7 new products/services, administrative techniques, operating technologies, etc.

typically adopts a very competitive, undo-thecompetitors posture.

In general, the top managers of my firm have...

RT1 a strong proclivity for low-risk proj
normal and certain rates of return).

In general, the top managers of my firm believe that...

owing to the nature of the environment, it is

owing to the nature of the environment, bold,

RT2 best to explore it gradually via timid, incremental behavior.

1 to 7 wide-ranging acts are necesary to achieve the firm's objectives.

When confronted with decision-making situations involving uncertainty, my firm...

typically adopts a cautious, wait-and-see

RT3 posture in order to minimize the probability 1 to 7 of making costly decisions. typically adopts a bold, aggressive posture in order to maximize the probability of exploiting potential opportunities.

Die Differenzierung eines Konstrukts nach einstellungs- oder verhaltensbezogenen Aspekten haben Sozialpsychologen intensiv erforscht (Ajzen 1991; Bagozzi 1992; Fazio 1990; Fishbein 1979; Olson/Zanna 1993), wobei eine Einstellung eine individuelle Objektbeurteilung abbildet (Ajzen/Fishbein 1977; Fazio et al. 1986). Eine Einstellung stellt eine Kombination aus dem Affekt (Gefühl), der Kognition (Überzeugungen und Ideen) und der Willenskraft (Absichten und Verhaltenstendenzen) dar (z. B. Kothandapani 1971; Shaver 1987). Objekte, zu denen Individuen eine bestimmte Einstellung haben, können Dinge, Personen, Ereignisse oder Tätigkeiten sein. Verhalten wird als eine oder mehrere beobachtbare Handlungen definiert (Ajzen/Fishbein 1977). Diese von Sozialpsychologen vorgenom- 
menen Unterscheidungen sind mit den zuvor erwähnten einstellungs- und verhaltensbasierten Perspektiven im Rahmen der EO-Skala vergleichbar.

Nach der „Theory of Planned Behavior“ sind Einstellungen und Verhaltensabsichten Voraussetzung für tatsächliches Verhalten (Ajzen 1991). Ebenso entstehen nach dem unternehmerischen Ereignismodell von Shapero und Krueger Neigung und die Absicht zu handeln vor dem tatsächlichen Verhalten (Krueger et al. 2000; Shapero 1982). Nach Bagozzi und Edwards (1998) führen Indikatoren, die innerhalb einer Skala Ursache oder Wirkung repräsentieren, zu verzerrten Messungen. Daher sollte, streng genommen, die Integration von sowohl einstellungs- als auch verhaltensbasierten Indikatoren in der Skala von unternehmerischer Orientierung vermieden werden. Sozialpsychologen sind sich einig, dass es zwischen Einstellungen und Verhaltensweisen keinen konsistenten Zusammenhang gibt (Ajzen/Fishbein 2005). Durchschnittliche Korrelationen zwischen Einstellungen und tatsächlichen Verhaltensweisen reichen von -0.20 bis 0.73 (Glasman/Albarracin 2006). Folglich ist nicht anzunehmen, dass Einstellungen tatsächliches Verhalten zuverlässig vorhersagen. Eine positive Einstellung gegenüber oder eine Bereitschaft zu unternehmerischer Tätigkeit führen nicht automatisch zu tatsächlichen unternehmerischen Handlungen. Die Integration von einstellungs- und verhaltensbasierten Indikatoren in der EO-Skala könnte demnach die Forschungsergebnisse verzerren.

Es ist daher zu klären, ob das EO-Konstrukt eine unternehmerische Disposition (d. h. eine Einstellung) oder eine unternehmerische Handlung (d. h. ein Verhalten) abbildet, wobei Evidenz für eine eher einstellungsbasierte Perspektive existiert. Erstens gehört EO zur konzeptionellen Familie der strategischen Orientierungen (vgl. Wiklund/Shepherd 2003), die als "guiding principles that influence a firm's marketing and strategy-making activities» verstanden werden (vgl. Noble et al. 2002, 25). Der Begriff 'Orientierung' umschreibt dabei eine allgemeine oder dauerhafte Ausrichtung von Gedanken, Neigungen oder Interessen (Covin/Lumpkin 2011). Basierend auf dieser Perspektive kann EO eher als eine Einstellung als ein Verhalten betrachtet werden. Zweitens hat die Studie von Wiklund (1998) gezeigt, dass das EO-Konstrukt nur zu 27\% unternehmerisches Verhalten erklärt. Drittens werden sowohl einstellungs- als auch verhaltensbasierte Indikatoren innerhalb der EO-Skala nicht als objektiv beobachtbare Indikatoren verstanden, sondern als von Managern subjektiv wahrgenommene Indikatoren. Nachdem jüngste Studien erklären, dass EO mit Bereitschaften oder Neigungen zu innovativen, proaktiven und risikoreichen Verhaltensweisen in Verbindung steht, entwickelte sich die einstellungsbasierte Perspektive schliesslich zum weitgehend akzeptierten Ansatz (z. B. Simsek et al. 2010).

Empfehlung 1: EO sollte als eine Management-Disposition definiert werden, die sich auf unternehmerische Tätigkeiten bezieht.

\subsubsection{Dimensionen von EO}

EO ist ein multidimensionales, komplexes Konstrukt und "a single theoretical concept», das aus "several distinct but related dimensions» besteht (Williams et al. 2003, 909). Obwohl das EO-Konstrukt meist durch die drei Dimensionen Innovativität, Risikobereitschaft und Proaktivität konzipiert wird, schlagen Autoren entweder zusätzliche (Covin/ Covin 1990; Lumpkin/Dess 1996) oder weniger Dimensionen (Knight 1997; Merz/Sauber $1995)$ vor. 
Abweichend von der originalen EO-Skala von Covin und Slevin (1989) werden als zusätzlich zu berücksichtigende Dimensionen Unabhängigkeit und kompetitive Aggressivität vorgeschlagen (Lumpkin/Dess 1996). Unabhängigkeit bezieht sich auf die «independent action of an individual or a team in bringing forth an idea or a vision and carrying it through to completion» (Lumpkin/Dess 1996, 140). Kompetitive Aggressivität bedingt "a firm's propensity to directly and intensely challenge its competitors to achieve entry or improve position, that is, to outperform industry rivals in the marketplace" (Lumpkin/Dess 1996, 148). Die Berücksichtigung dieser beiden Dimensionen im Rahmen der Verwendung der originalen EO-Skala von Covin und Slevin (1989) erscheint jedoch sowohl theoretisch als auch methodisch problematisch.

Aus theoretischen Überlegungen könnte man argumentieren, dass Unabhängigkeit und kompetitive Aggressivität nicht Merkmale einer unternehmerischen Orientierung sind. Zum einen bezieht sich Unabhängigkeit auf Individuen oder Teams innerhalb einer Unternehmung, was zur Annahme führt, dass sich Unabhängigkeit nicht als Dimension eines Konstrukts auf Unternehmensebene eignet. Lumpkin et al. (2009) betonen, dass unabhängige Individuen oder Teams ausschlaggebend für die Förderung unternehmerischen Verhaltens auf Unternehmens- oder Abteilungsebene sind. Obwohl einige Autoren dieser These zustimmen (Antoncic/Hisrich 2003; Kanter et al. 1990), handelt es sich eher um einen kontextspezifischen Aspekt, der unternehmerisches Verhalten ermöglicht und nicht um eine notwendige Eigenschaft einer unternehmerisch ausgerichteten Organisation (Kuratko et al. 2005). Unabhängigkeit "provides the impetus needed", "fosters", "encourages" und bedingt damit EO (Lumpkin et al. 2009, 48-49). Dennoch ist Unabhängigkeit keine notwendige Charakteristik, dass eine Organisation unternehmerisch geführt gilt (Davis 2007).

Zum anderen wird kompetitive Aggressivität zumindest teilweise durch die Dimension Proaktivität respräsentiert. Proaktivität ist "characterized by the introduction of new products and services ahead of the competition" (Rauch et al. 2009, 763). Lumpkin und Dess (1996) unterscheiden Proaktivität eindeutig von kompetitiver Aggressivität und behaupten, dass Unternehmungen proaktiv handeln können ohne dabei mit Wettbewerbern zu konkurrieren. Proaktivität und kompetitive Aggressivität umfassen die Konfrontation mit Wettbewerbern, jedoch ergreifen proaktive Unternehmen Marktchancen als Erstes. Das Ziel kompetitiver Aggressivität besteht darin, gefährliche Konkurrenten leistungsmässig zu übertreffen (Lumpkin/Dess 1996). Empirisch gesehen sind sich die Operationalisierungen der beiden Dimensionen zu ähnlich. Lumpkin und Dess (2001) messen kompetitive Aggressivität mit zwei Indikatoren. Eines davon stammt aus der originalen Proaktivitätsskala von Covin und Slevin (1989) (Indikator PRO3, vgl. Tabelle 2). Covin und Covin (1990) messen kompetitive Aggressivität mit derselben Skala, die sie in einer vorhergehenden Studie für die Messung von Proaktivität verwendeten (vgl. Covin/Slevin 1989). Abgesehen davon stellen Autoren infrage, ob kompetitive Aggressivität, wie sie von Lumpkin und Dess (1996) definiert wird, per se eine Eigenschaft einer unternehmerisch orientierten Organisation darstellt. Konkurrenten herauszufordern wird zwar als bestimmende Facette von kompetitiver Aggressivität angesehen, jedoch nicht als unternehmerische Eigenschaft (Davis 2007).

Aus methodischer Perspektive hat die Änderung der ursprünglichen EO-Konzeptualisierung durch Lumpkin und Dess (1996) zu der unerwünschten Situation geführt, dass das Konzept 'gedehnt' wurde (vgl. George/Marino 2011). Demnach erhöhen die Autoren die definierenden Konstrukteigenschaften, während sie versuchen, die Anzahl der Fälle in de- 
nen ein Unternehmen als unternehmerisch orientiert gilt, aufrecht zu erhalten oder zu erhöhen (Collier/Mahon 1993; Satori 1984). Lumpkin und Dess (1996) haben zwei Eigenschaften zu den drei originalen Dimensionen Innovativität, Proaktivität und Risikobereitschaft hinzugefügt. Die zugrundeliegenden theoretischen Argumente, welche Firmen als unternehmerisch orientiert gelten, unterscheiden sich stark von der ursprünglichen EOKonzeptualisierung von Covin und Slevin (1989). Bezeichnet man beide Konzeptualisierungen als 'Entrepreneurial Orientation', dann wird impliziert, dass diese den gleichen konzeptionellen Bereich abdecken (vgl. George/Marino 2011). Das originale EO-Konstrukt wurde mit drei Dimensionen erfasst. Verglichen mit der fünfdimensionalen Konzeptualisierung von Lumpkin und Dess (1996) beschreibt die originale Definition allgemeiner, was eine unternehmerisch geführte Firma ausmacht. Die Konzeptualisierung von Lumpkin und Dess (1996) ist konzeptionell spezifischer und impliziert weniger Organisationen, die als unternehmerisch orientiert gelten (George/Marino 2011). Der Definitionsbereich des Konstrukts wird durch die Konzeptualisierung von Lumpkin und Dess (1996) demnach anders interpretiert.

Empfehlung 2: Das EO-Konstrukt sollte durch die drei Dimensionen Innovativität, Proaktivität und Risikobereitschaft abgebildet werden.

\subsection{Der Geltungsbereich des EO-Konstrukts}

Konstrukte in der betriebswirtschaftlichen Forschung sind nicht universell einsetzbar. Deshalb beschreibt das Kriterium des Geltungsbereichs („scope condition“) die “contextual circumstances under which a construct will or will not apply" (Suddaby 2010, 347). Dies ist insbesondere wichtig, da ,a lack of clarity often makes it difficult to distinguish the focal concept from other similar concepts in the field" (Podsakoff et al. 2016). Der Geltungsbereich eines Konstrukts sollte Aspekte wie Raum, Zeit und Wert erfassen (Bacharach 1989).

Zwar wurde in bestehenden Studien der Geltungsbereich von EO implizit adressiert, jedoch konnte bisher kein explizit formulierter Geltungsbereich identifiziert werden. Eine theoretische Grundlage leitet die Bestimmung des Geltungsbereichs eines Konstrukts (Suddaby 2010). Diese theoretische Untermauerung fehlt dem EO-Konstrukt (Covin/Lumpkin 2011). Wir beziehen mehrere unserer Empfehlungen hinsichtlich des Geltungsbereichs des Konstrukts exemplarisch auf die theoretische Perspektive der 'Dominant Logic' (Covin/ Slevin 2002; Meyer/Heppard 2000; Prahalad/Bettis 1986). Eine EO dominante Logik entsteht, wenn Einstellungen und Verhaltensweisen einer Unternehmung und derer Mitglieder in Richtung Unternehmertum ausgerichtet ist. Dieser Blickwinkel wurde von Covin und Lumpkin (2011) als theoretische Perspektive für die EO-Forschung vorgeschlagen.

\subsubsection{Der räumliche Geltungsbereich}

Der räumliche Geltungsbereich eines Konstrukts beeinflusst dessen Anwendbarkeit auf unterschiedlichen Analyseebenen, Organisationstypen und Kulturen (Suddaby 2010).

Die Anwendung auf unterschiedlichen Analyseebenen. Typischerweise werden Konstrukte mit konkreten Analyseebenen verbunden (Klein et al. 1994). Da EO ursprünglich als ein Konstrukt auf Unternehmensebene konzipiert wurde (Covin et al. 2006; Covin and Slevin 1989; Rauch et al. 2009; Zahra 1993), haben es nur wenige Forschende für Analysen auf individueller Ebene eingesetzt (z. B. Dickson/Weaver 1997; Kollmann et al. 2007). 
EO wird als ein Konstrukt auf Unternehmens- bzw. auf Abteilungsebene (wenn es um Unternehmen mit mehreren Abteilungen geht) betrachtet (vgl. Green et al. 2008). Indikatoren INN1, RT1 und RT2 (vgl. Tabelle 2) fragen nach der Einstellung von Top Managern, während sich die anderen Indikatoren auf Einstellungen und Verhaltensweisen der gesamten Unternehmung beziehen. Die Einstellungen von Top Managern sind zwar massgeblich für die gesamte Unternehmung, unterscheiden sich jedoch häufig von denen der restlichen Belegschaft. Wiklund und Shepherd $(2003,1310)$ merken an, dass insbesondere "in large firms CEOs might be separated from 'how a firm operates' by layers of middle managers". Dennoch wird die EO-Skala von Top Managern bewertet (Lyon et al. 2000).

Aus der Perspektive der 'Dominant Logic' besteht eine dominante Logik dann, wenn unternehmerische Einstellungen und Handlungen innerhalb der Unternehmung konsistent sind (Meyer/Heppard 2000). Insbesondere sind drei Analyseebenen zu berücksichtigen: die Unternehmung als Organisationseinheit, das Top Management und die individuellen Organisationsmitglieder (Covin/Slevin 2002; Ireland et al. 2009). Bei der Angleichung unternehmerischer Werte, Einstellungen und Handlungen nimmt das Top Management eine wesentliche Rolle ein (Meyer/Heppard 2000; Prahalad/Bettis 1986). Covin und Slevins (1989) Konzeptualisierung von EO als Reflektion des Top Managements ist somit adäquat.

Empfehlung 3: EO sollte als ein von Top Managern wahrgenommenes Phänomen auf Unternehmensebene betrachtet werden.

Die Anwendung für unterschiedliche Organisationstypen. Gemäss den Ursprüngen von EO und Millers (1983) Auffassung, ist das EO-Konstrukt insbesondere für die Analyse etablierter Unternehmungen anwendbar (Covin et al. 2006). Obwohl EO-Forschung zumeist mit Bezug auf etablierte kleine und mittlere Unternehmungen stattfindet (z. B. Covin et al. 2006; Hansen et al. 2011; Lumpkin/Dess 2001; Richard et al. 2004; Wiklund 1999) schlagen Autoren vor, das Konstrukt auch im Kontext von neu gegründeten, börsennotierten, Non-Profit- oder Grossunternehmungen anzuwenden (z. B. Covin/Wales 2012; Morris/Paul 1987). Die Begründung, dass EO für viele verschiedene Organisationstypen wichtig ist, liegt an der allgemeinen Verkürzung von Produkt- und Geschäftsmodellzyklen ( $\mathrm{Ha}$ mel 2000). Dies betrifft die meisten Unternehmungen und daher ist eine EO notwendig, um stetig neue Geschäftsmöglichkeiten zu identifizieren (Wiklund/Shepherd 2005).

Eine sinnvolle Anwendung des Konstrukts in gemeinnützigen Unternehmungen ist aufgrund fehlender Absicht zur Gewinnmaximierung sowie geringerer Bereitschaft zu innovativen, proaktiven und risikobehafteten Handlungen eingeschränkt.

Empfehlung 4: EO sollte als ein Phänomen betrachtet werden, das in gewinnorientierten Start-Ups, kleinen, mittleren und etablierten Unternehmungen beobachtbar ist.

Die Anwendung in unterschiedlichen Kulturen. Das EO-Konstrukt stammt ursprünglich aus dem U.S. amerikanischen Kontext. Eine valide Anwendung der EO-Skala in einem anderen nationalen Kontext erscheint fraglich. Analysen und Rückschlüsse von EO-Daten, die in nicht amerikanischen Kontexten erhoben wurden, sind "at best ambiguous and at worst erroneous" (Steenkamp/Baumgartner 1998, 78). Ob das Konstrukt nur in spezifischen kulturellen Kontexten oder allgemein anwendbar ist, kann man laut Wickliffe (2004) anhand der Konsistenz der Reliabilitätsmessungen des Konstrukts, d. h. anhand der Alphakoeffizienten der unterschiedlichen Stichproben sehen. Die Reliabilitätswerte lie- 
gen in länderübergreifenden Studien und in Studien, die in einem nicht-amerikanischen Land durchgeführt wurden, überwiegend oberhalb der Grenze von .70 (Nunnally 1978). Daraus ist nicht zu schliessen, dass EO ein global anwendbares Konstrukt ist, da verschiedene Effekte gegen die Verallgemeinbarkeit der Messung sprechen können (vgl. Rossiter 2002). Ein Beispiel hierfür ist eine fehlende Konstruktäquivalenz zwischen den Ländern, d. h. ob die Bewertung kontextspezifisch ident ist oder Begriffe semantisch gleich interpretiert werden (Singh 1995). Zudem bedeuten Abweichungen der Reliabilitätswerte von EO in unterschiedlichen Stichproben nicht automatisch, dass das Konstrukt nicht in nichtamerikanischen Kontexten angewandt werden kann. Abweichungen können auch bei Ergebnissen mit lokalem Bezug auftreten.

Aufgrund unterschiedlicher kultureller Werte und Normen, die in mentalen Prozessen der Menschen oder in organisationalen und staatlichen Strukturen verankert sind, können Phänomene wie EO nicht in jedem Land gleichermassen beobachtet und interpretiert werden (Hampden-Turner/Trompenaars 2006; Hofstede 1993). Empirische Studien von Knight (1997), Wiklund (1999), Brown et al. (2001), Kreiser et al. (2002) sowie Arbaugh et al. (2009) belegen, dass das Konstrukt in nicht amerikanischen Kontexten anwendbar ist. Kemelgor (2002) und Antoncic and Hisrich (2001) stellen die länderübergreifende Anwendung des Konstrukts jedoch in Frage. Hansen et al. (2011) haben anhand von konfirmatorischen Multigruppenmodellen die Validität der EO-Skala in sieben Ländern untersucht, indem nicht nur länderübergreifende Abweichungen von Reliabilitätswerten, sondern unterschiedliche länderübergreifende Invarianzen analysiert wurden. Die Forschungsergebnisse zeigen, dass EO länderübergreifend ein multidimensionales Konstrukt mit denselben Dimensionen (Innovativität, Proaktivität und Risikobereitschaft) ist. Dabei werden jedoch zwei Indikatoren der Skala von Covin und Slevin (1989) (INN2 und PRO1) in verschiedenen Ländern unterschiedlich interpretiert. Dieses Ergebnis zeigt, dass Forschende vorsichtig sein müssen, wenn sie EO-Messungen unterschiedlicher Länder vergleichen.

Divergierende Forschungsergebnisse der länderübergreifenden EO-Analysen weisen darauf hin, dass die Interpretation von Indikatoren von Land zu Land unterschiedlich sein kann. Innovative, proaktive und risikobereite Einstellungen und Verhaltensweisen können je nach Kontext unterschiedlich verstanden und bewertet werden (z. B. Hansen et al. 2011).

Empfehlung 5: EO sollte als ein Konstrukt angesehen werden, das im amerikanischen Kontext bestmöglich anwendbar ist.

\subsubsection{Der zeitliche Geltungsbereich}

Der zeitliche Geltungsbereich für das EO-Konstrukt wurde in bisheriger Forschung noch nicht explizit definiert. Die Auffassung, dass das Konstrukt eine Einstellung darstellt (Wiklund/Shepherd 2005) zeigt jedoch, dass sich eine EO im Zeitablauf (längerfristig) entwickelt und eine unternehmensweite Integration zeitaufwändig ist. Dies steht auch im Einklang mit der Perspektive der Dominant Logic, nach der fortwährende und dauerhafte Überzeugungen, Einstellungen und Philosophien charakteristisch für unternehmerisch orientierte Firmen sind (Covin/Lumpkin 2011). Gleichzeitig erfordert unternehmerisches Verhalten im Sinne einer EO strategische Flexibilität, Agilität sowie Anpassungsfähigkeit und stellt ein dynamisches Phänomen dar (Covin/Slevin 2002; Kuratko/Audretsch 2009). 
Empfehlung 6: EO sollte als ein langfristiges Phänomen betrachtet werden, das nur schrittweise verändert werden kann.

\subsubsection{Der Geltungsbereich in Bezug auf Wertvorstellungen}

Der Geltungsbereich eines Konstrukts bezogen auf Wertvorstellungen ist "a result of the assumptions or world view of the researcher" (Suddaby 2010, 350). Die meisten Konstrukte im Bereich der strategischen Unternehmensführung nehmen die Perspektive von Top Managern ein. Während EO als ein Konstrukt auf Unternehmensebene angesehen wird, betonen auch Covin und Slevin (1989) die Wahrnehmung der Top Manager. Aus der Perspektive der Dominant Logic sollte EO über alle Hierarchien einer Unternehmung kohärent sein (Covin/Slevin 2002; Meyer/Heppard 2000). Auch diese Perspektive betont die Rolle des Top Managements bei der Angleichung von unternehmerischen Werten, Einstellungen und Handlungen. Zudem beziehen sich wertbezogene Annahmen auch auf die Substanz und das Ausmass der Wertgenerierung. Steigert EO den Unternehmenserfolg beispielsweise bezogen auf die Produktivität, den Gewinn oder das Wachstum oder leistet EO im weiteren Sinne einen Beitrag zum Shareholder Value, zum Stakeholder Value oder sogar zu gesellschaftlichen Werten? Covin und Slevin $(2002,310)$ argumentieren basierend auf der Perspektive der Dominant Logic, dass "an entrepreneurial dominant logic should facilitate an organization's ability to create long-term shareholder wealth".

Empfehlung 7: EO sollte als ein unternehmensweites Phänomen betrachtet werden, das durch das Top Management entwickelt wird und auf den langfristigen Wohlstand der Eigentümer abzielt.

\subsection{Zusammenhänge zwischen EO und anderen Konstrukten}

"Constructs exist only in referential relationships, either explicit or implicit, with other constructs and with the phenomena they are designed to represent" (Suddaby 2010, 350). Folglich erfordert Konstruktschärfe, dass Forschende die theoretischen Zusammenhänge mit anderen, unterscheidbaren Konstrukten aufzeigen. Diese durch Argumente gestützten theoretischen Beziehungen stellen die Grundlage für eine Theorie dar (Bacharach 1989, Podsakoff et al. 2016). Obwohl zahlreiche Studien Zusammenhänge zwischen EO und anderen Konstrukten testen, wurde die EO-Forschung bisher nur selten in wissenschaftlich anerkannte Theorien eingebettet (Covin/Lumpkin 2011).

\subsubsection{Erforschte Zusammenhänge bestehender EO-Forschung}

Bestehende (empirische) Erkenntnisse der Beziehung zwischen EO und dem Unternehmenserfolg beruhen primär auf Analysen linearer funktioneller Zusammenhänge, obwohl auch nicht-lineare Zusammenhänge geprüft wurden (z. B. Tang et al. 2008). Der positive Zusammenhang zwischen den Konstrukten wird durch das Potential unternehmerisch orientierter Organisationen begründet, sich auf hochwertige Marktsegmente zu fokussieren. Dadurch gelingt eine Positionierung als Pionierunternehmung; höhere Preise können gesetzt und damit höhere Gewinne erzielt werden (Covin/Slevin 1991; Wiklund 1999; Zahra/Covin 1995). Da EO in seiner Beziehung zum Unternehmenserfolg als ein kontextspezifisches Konstrukt angesehen wird, wurde die Beziehung zwischen EO und dem Unternehmenserfolg unter Berücksichtigung von Moderatoren untersucht. Moderatoren können in 
umweltbedingte (externe) und organisationale (interne) Variablen eingeteilt werden (Lumpkin/Dess 1996; Wiklund/Shepherd 2005). Bei vielen dieser Studien sind signifikante und positive Zusammenhänge zwischen EO, den Moderatoren und dem Unternehmenserfolg erkannt worden. Dennoch wurden auch nicht signifikante oder negative Beziehungen zwischen den beiden Konstrukten nachgewiesen (Rauch et al. 2009). Diese inkonsistenten Ergebnisse könnten auf abweichende Konzeptualisierungen von EO zurückzuführen sein. Die Beziehungen zwischen EO und anderen Konstrukten unterscheidet sich nicht nur hinsichtlich der Indikatoren, die für die Konstruktmessung verwendet werden, sondern auch hinsichtlich der Definition des Konstrukts als eindimensional oder mehrdimensional (Law et al. 1998).

\subsubsection{Das theoretische Fundament der EO-Forschung}

Während innerhalb der EO-Forschung Ergebnisse existieren, welche die Beziehung von EO $\mathrm{zu}$ anderen Konstrukten untersuchen, ist die theoretische Fundierung der Erkenntnisse häufig rudimentär (Covin/Lumpkin 2011). Die theoretische Begründung der Forschungskonzeption erfolgt nicht immer explizit. Stattdessen wird eine fundierte Begründung der Beziehung zwischen Konstrukten mit der Enumeration des Literaturstromes verwechselt.

Autoren haben vorgeschlagen, EO-Forschung in den theoretischen Rahmen der Dominant Logic (Prahalad/Bettis 1986) bzw. der Entrepreneurial Dominant Logic (Meyer/ Heppard 2000) einzubetten. Die Dominant Logic stellt darauf ab, inwiefern Unternehmungen "conceptualize the business and make critical resource allocation decisions" (Prahalad/Bettis 1986, 490). Die theoretischen Ausführungen beziehen sich dabei auf die kollektive Bestimmung sowie den Umfang von unternehmerischen Routinen. Im Rahmen der Entrepreneurial Dominant Logic ist die Denkweise einer Unternehmung hinsichtlich der Wahrnehmung, dem Ausnutzen und der Erforschung von Möglichkeiten auf die Wertschöpfung fokussiert (Meyer/Heppard 2000).

Zwar wurde die Perspektive der (Entrepreneurial) Dominant Logic als theoretische Grundlage für die Erklärung der Beziehung von EO zu anderen Konstrukten betont, die Perspektive jedoch in empirischer Forschung bisher nicht explizit konzeptionalisiert.

Die Dominant Logic einer Unternehmung umfasst im Wesentlichen das "unternehmerische Denkmodell“. Eine vorherrschende Entrepreneurial Dominant Logic existiert, wenn eine Unternehmung "and its members interpret, value, and act on information on the basis of the potential of value creation and profitability for the firm" (Meyer/Heppard 2000, 2). Eine Entrepreneurial Dominant Logic äussert sich in einer dauerhaften unternehmerisch orientierten Haltung (EO) und in dynamischen, unternehmerisch orientierten Routinen (unternehmerisches Verhalten) (vgl. Covin/Lumpkin 2011; Covin/Slevin 2002). Unternehmungen, die durch eine Entrepreneurial Dominant Logic charakterisiert werden, sind fähig, sich unternehmensweit konsistent auszurichten („alignment“) und können sich bei Bedarf flexibel anpassen („adaptability“) (Covin/Lumpkin, 2011; Covin/Slevin, 2002). Die Fähigkeit der konsistenten Anpassung bezieht sich dabei auf eine kohärente Ausnutzung aufkommender Marktchancen. Die Fähigkeit der flexiblen Anpassung bezieht sich auf die dynamische Implementierung veränderter Anforderungen. Die Notwendigkeit zur Veränderung kann sowohl auf Informationen aus dem Umfeld zurückzuführen sein als auch auf Erkenntnisse, die im Rahmen der Erforschung neuer Marktchancen gewonnen werden (Kuratko/Audretsch 2009; Obloj et al. 2010). 
Die Entrepreneurial Dominant Logic Perspektive bietet eine geeignete theoretische Grundlage für die Erforschung von Beziehungen zwischen EO und anderen Konstrukten. Diese theoretische Perspektive könnte Grundlage für die Beantwortung folgender Fragen sein: Weisen erfolgreiche Unternehmungen eine Entrepreneurial Dominant Logic auf? Welchen Einfluss hat die Verbreitung von EO innerhalb einer Unternehmung? Inwiefern können Top Manager Spannungen bewältigen, die auf Anpassungen zurückzuführen sind?

Empfehlung 8: Basierend auf der theoretischen Perspektive der Entrepreneurial Dominant Logic sollte EO als eine dauerhafte unternehmerische Einstellung betrachtet werden, die über alle Hierarchien im Unternehmen hinweg existiert und zu dynamischen, unternehmerischen Handlungen führt.

\subsection{Die Kohärenz des EO-Konstrukts}

Mit Bezug auf die Kohärenz eines Konstrukts wird festgestellt, ob "the various attributes of a phenomenon are adequately contained within a construct that is, do these attributes hang together in a logical and empirically convincing way?" (Suddaby 2010, 352). Die Kohärenz zwischen EO und dessen Beziehung zu zugrundeliegenden Dimensionen betrifft vor allem Frage ob das Konstrukt tatsächlich durch dessen Indikatoren reflektiert wird oder ob die Indikatoren das EO-Konstrukt formen (d. h. die Frage, ob das Konstrukt als reflektiv oder formativ modelliert werden soll) (z. B. George 2011). Ausserdem ist zu klären, ob EO als eindimensionales oder mehrdimensionales Konstrukt konzeptionalisiert wird.

\subsubsection{Reflektive versus formative Messung}

Die Frage, ob ein Konstrukt auf reflektive oder formative Weise gemessen werden soll, basiert auf der Beziehung des Konstrukts selbst zu dessen Indikatoren (Diamantopoulos et al. 2008; Edwards/Bagozzi 2000; Jarvis et al. 2003; Podsakoff et al. 2006). Im Fall der reflektiven Messung stellen Indikatoren eine "reflection or manifestation of the underlying construct" dar (Podsakoff et al. 2006, 199). Ein solches Konstrukt wird als latente Variable bezeichnet (MacCallum/Browne 1993; Podsakoff et al. 2006). Im Gegensatz dazu stellen Indikatoren im Falle einer formativen Messung die Ursache für ein Konstrukt dar. Das Konstrukt stellt also eine Zusammensetzung aus dessen Indikatoren dar, welche als definierende Eigenschaften gelten (MacCallum/Browne 1993; MacKenzie et al. 2005; Podsakoff et al. 2006). Es ist wichtig zu spezifizieren, ob ein Konstrukt in reflektiver oder formativer Weise gemessen werden soll, da Fehlspezifikationen das Risiko von Fehlern der Typen I und II erhöhen (Jarvis et al. 2003; MacKenzie et al. 2005) und eine bedeutungsvolle Theorietestung damit unmöglich ist (Edwards/Bagozzi 2000). Obwohl EO in bisherigen Studien nahezu durchgehend als ein reflektives Konstrukt angesehen wurde, ist diese Art der Messung diskussionswürdig (Anderson et al. 2015; George 2011). Dies ist insbesondere auf teils unvereinbare Konstruktdefinitionen zurückzuführen. Eine der ältesten Definitionen von EO beschreibt, dass "we can tentatively view entrepreneurship as a composite weighting" der Dimensionen Innovativität, Proaktivität und Risikobereitschaft (Miller 1983, 771). Diese Definition beschreibt EO als eine "Zusammensetzung" und schlägt damit eine formative Messung des Konstrukts vor, denn "formative constructs are a composite of multiple measures" (vgl. Anderson et al. 2015; Covin et al. 2006; Petter et al. 2007, 624). EO "is revealed through an organization's exhibition of risk taking, inno- 
vativeness, and proactiveness" (Covin et al. 2006, 57). Diese Definition verdeutlicht durch die Beschreibung „is revealed through“, d. h. dass EO durch dessen Dimensionen offenbart wird, eine reflektive Messung.

Die Modellierung von EO als reflektives oder formatives Konstrukt betrifft auch die Frage, ob dessen Indikatoren Einstellungen oder Verhaltensweisen darstellen. Die Originalskala von Covin und Slevin (1989) weist eine Mischung von einstellungs- und verhaltensbasierten Indikatoren auf. Weiter oben haben wir den Gedanken, dass EO eher eine Einstellung als ein Verhalten darstellt, näher ausgeführt. Einstellungen werden im Allgemeinen als reflektiv modelliert, da sie ein Ausdruck des zugrundeliegenden Konstrukts sind. Gemäss der einstellungsbasierten Perspektive ist EO eine strategische Orientierung, die stark im Selbstverständnis einer Unternehmung verwurzelt ist und sich durch Innovativität, Proaktivität und Risikobereitschaft äussert (Coltman et al. 2008).

Empfehlung 9: Covin und Slevins (1989) EO-Konstrukt betrachtet als eine Management-Disposition, die für unternehmerische Tätigkeiten steht, sollte als reflektives Konstrukt modelliert werden.

\subsubsection{Dimensionalität}

Die Begründer des EO-Konstrukts Miller (1983) sowie Covin und Slevin (1989) haben EO anhand der Dimensionen Innovativität, Proaktivität und Risikobereitschaft konzeptionalisiert. Diese Konzeptualisierung impliziert ein multidimensionales EO-Konstrukt, denn "it consists of a number of interrelated attributes or dimensions" (Law et al. 1998, 741). Multidimensionale Konstrukte können als latente Modelle, aggregierte Modelle oder Profil-Modelle abgebildet werden (Law et al. 1998). Covin und Slevin (1989) brachten hervor, dass EO ein aggregiertes Modell darstellt, da die neun Indikatoren, die mit den Dimensionen Innovativität, Proaktivität und Risikobereitschaft verbunden sind, gemeinsam das Konstrukt repräsentieren. Lumpkin und Dess (1996) argumentieren, dass die einzelnen Dimensionen den Unternehmenserfolg unterschiedlich beeinflussen können. Viele Forschende teilen diese Ansicht. Sie haben die Dimensionen separat betrachtet und argumentiert, dass eine Unternehmung auch dann als unternehmerisch orientiert gilt, wenn nur eine Dimension von EO stark ausgeprägt ist (z. B. Kreiser et al. 2002; Stetz et al. 2000). Dies steht im Gegensatz zur originalen Konzeption des Konstrukts, demzufolge eine unternehmerische Orientierung nur dann existiert, wenn alle Dimensionen gleichzeitig vorhanden sind (Covin/Wales 2012). Wie eine Metaanalyse zum Zusammenhang von EO und dem Unternehmenserfolg zeigt, dominiert diese aggregierte Perspektive noch immer (Rauch et al. 2009). Die Entscheidung, ob ein Konstrukt in aggregierter Form oder bezogen auf dessen einzelne Dimensionen analysiert wird, hängt im Wesentlichen vom Fokus des Forschenden und "the generality or specificity of one's theoretical interest" ab (MacKenzie et al. 2005, 713). Das Argument, dass die EO-Dimensionen separat analysiert werden müssen weil sich die einzelnen Dimensionen voneinander unterscheiden können (Lumpkin/Dess 1996), ist dagegen fragwürdig. Per Definition repräsentiert tatsächlich nur die gemeinsame Varianz das EO-Konstrukt, wohingegen einzelne Varianzen mit einem Messfehler einhergehen (Covin/Lumpkin 2011; Law/Wong 1999). Es ist also weiterhin fraglich, ob die Dimensionen Innovativität, Proaktivität und Risikobereitschaft geeignet sind "to be conceptualized under an overall abstraction" von EO (Law et al. 1998, 741; vgl. Suddaby 2010). 
Entscheiden sich Autoren auf theoretischer Basis, dass die drei Dimensionen zusammen das EO-Phänomen bilden wie von Miller (1983) sowie Covin und Slevin (1989) vorgeschlagen, eignet sich eine aggregierte Messung. Werden die einzelnen Dimensionen jedoch unabhängig voneinander analysiert, untersuchen Autoren ausschliesslich die Effekte von Innovativität, Proaktivität und Risikobereitschaft. Gemäss der originalen Konzeption von EO bedeutet dies jedoch, dass Autoren nicht das EO-Konstrukt messen, denn die originale Definition erklärt, dass sich EO durch die gleichzeitige Erscheinung der drei Dimensionen äussert (Covin/Slevin 1989). Forschende, die EO messen möchten, sollten sich demnach auf die gemeinsame Varianz der einzelnen Konstruktdimensionen fokussieren.

Empfehlung 10: EO sollte als ein aggregiertes, mehrdimensionales Konstrukt betrachtet werden.

\section{Zusammenfassung und Ausblick}

Die Einführung des EO-Konstrukts in die Literatur Mitte der 1970er Jahre stellt einen Meilenstein dar. Aktuell zählt EO zu den zentralen Konstrukten innerhalb der Management- und Entrepreneurship-Forschung. Divergierende Auffassungen zu dessen Konzeptualisierung und Operationalisierung geben Anlass zur kritischen Reflektion der Konstruktschärfe von EO. Diese divergierenden Perspektiven betreffen die Dimensionalität des EO-Konstrukts, die Eignung der konzeptualisierten Dimensionen (z. B. Lumpkin/Dess 1996), die Operationalisierung durch eine Mischung aus einstellungs- und verhaltensbasierten Indikatoren (z. B. Miller 2011; Wiklund 1999) und die Konzeptualisierung als reflektiv oder formativ (z. B. George 2011). Verschiedene Auffassungen dieser Punkte haben zur Folge, dass EO unterschiedlich definiert, interpretiert und angewendet wird. Eine gemeinsame, unwidersprüchliche Sprache innerhalb der Forschungsgemeinschaft ist kaum zu erkennen und behindert Forscherinnen und Forscher darin, gegenseitig auf ihren Ergebnissen aufzubauen und den Forschungsbereich weiterzuentwickeln. Auf Basis der Kriterien für Konstruktschärfe von Suddaby (2010), die ausschlaggebend sind für eine einheitliche Sprache einer Forschungsgemeinschaft, sind wir systematisch der Frage nachgegangen, wie wir den Unklarheiten des EO-Konstrukts begegnen könnten. Tabelle 3 zeigt einen Überblick über Kriterien der Konstruktschärfe, entsprechende Unklarheiten in der Anwendung von $\mathrm{EO}$ sowie daraus abgeleitete Empfehlungen. 


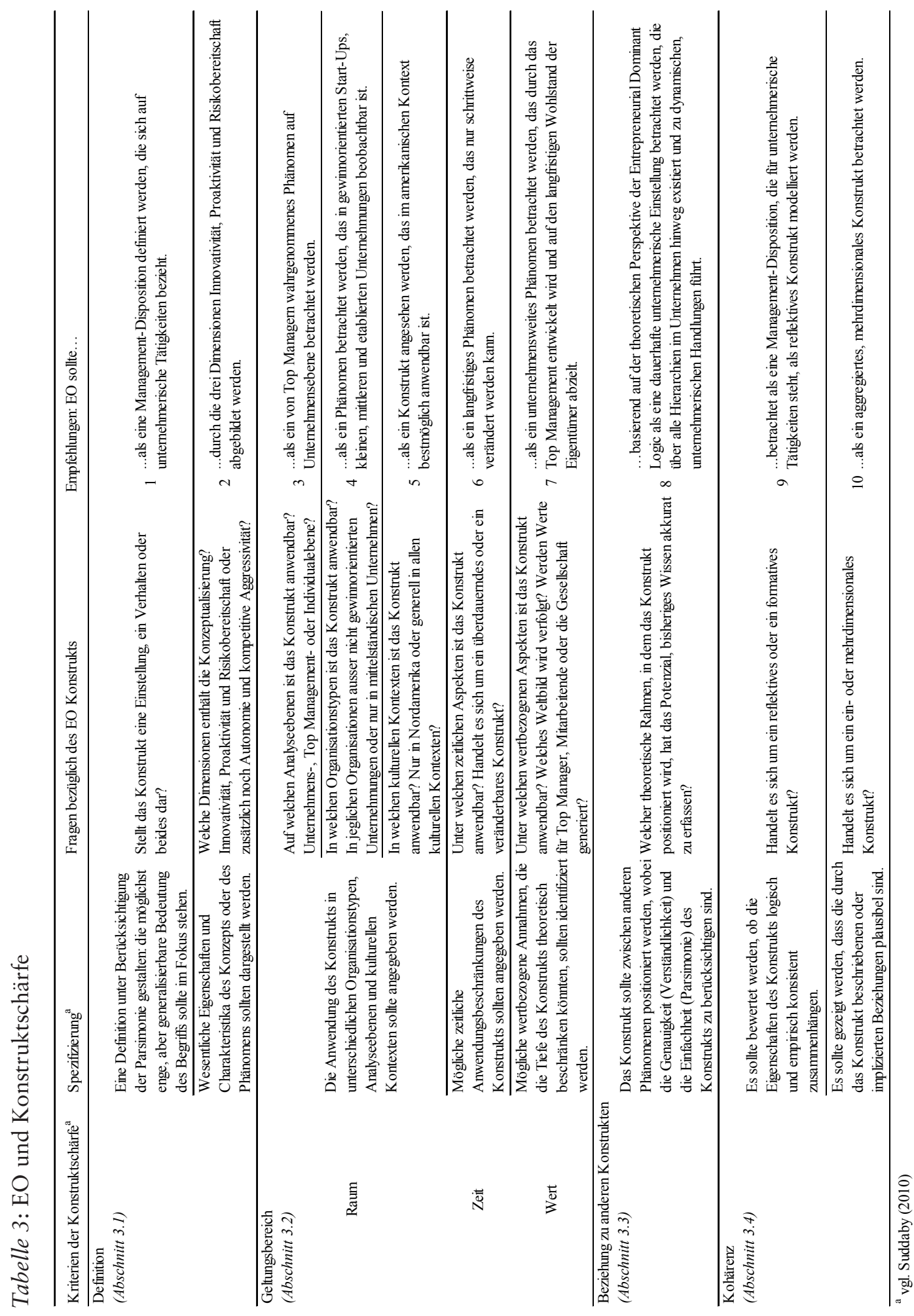


Wir stellen zwei elementare Aspekte in den Vordergrund, die beitragen können, die Unklarheiten des EO-Konstrukts zu reduzieren. Erstens sollten Forschende sich auf eine eindeutige Konzeptualisierung von EO einigen (Covin/Lumpkin 2011). Basierend auf unseren Ausführungen bestehender Diskussionen über das Konstrukt sowie der Kriterien für Konstruktschärfe haben wir zehn Empfehlungen zur Konzeptualisierung des Konstrukts aufgestellt (vgl. Tabelle 3). Bestehende Definitionen des EO-Konstrukts sind uneinheitlich (vgl. Tabelle 1). Eine umfassende Konstruktdefinition sollte Informationen über das im Fokus stehende Konstrukt enthalten, einschliesslich dessen Komponenten, Eigenschaften und Bewertungseinheiten (Rossiter 2002).

Zweitens sollten Autoren unterscheiden, ob EO eine Disposition ist, sich auf unternehmerisches Verhalten einzulassen, oder tatsächliches unternehmerisches Verhalten repräsentiert. Gemäss der Theory of Planned Behavior (Ajzen 1991) ist die Unterscheidung insofern wichtig, als Verhaltensdispositionen eine Voraussetzung für tatsächliches Verhalten darstellen. Einige Autoren untersuchen EO als ein verhaltensbezogenes Konstrukt, was letztlich zu einer schwachen theoretischen Argumentation der Hypothesen führt. Das Überwinden der Unklarheiten des EO-Konstrukts bedeutet demnach, dass Forschenden neben einer angemessenen Konzeptualisierung Alternativen für die Messungen von unternehmerischem Verhalten bereitstehen sollten.

Daneben haben wir dargelegt, wie Autoren EO künftig in die Theorie der Dominant Logic einbetten könnten. Insbesondere könnten auf Basis dessen z.B. folgende Fragen adressiert werden: Welche umweltbedingten, organisationalen und sozialen Kontexte fördern Entrepreneurial Dominant Logic? Wie kann eine unternehmensweite Implementierung von EO erreicht werden? Ist EO ein träges oder ein dynamisches Phänomen? Was sind die Folgen von EO und unternehmerischem Verhalten auf unterschiedlichen Analyseebenen (z. B. Top Management oder Individualebene)?

Schliesslich sollten Autoren des Forschungsbereichs Entrepreneurship auf Unternehmensebene den konzeptionellen Herausforderungen seiner Kernkonstrukte begegnen, um auf bisher angesammeltem Wissen aufzubauen und neues Wissen in diesem Forschungsfeld zu generieren. Um eine gemeinsame Basis für die Theorieentwicklung zu liefern, haben wir die Unklarheiten für das von Covin und Slevin (1989) operationalisierte EO-Konstrukt identifiziert und analysiert.

Insgesamt ist die hohe Bedeutung des EO-Konstrukts in der Management- und Entrepreneurship-Forschung unbestritten, und durch die Einführung von EO in die akademische Diskussion Mitte der 1970er Jahre wurde ein wesentlicher Meilenstein gelegt. Im Forschungsbereich sind in den letzten Jahrzehnten signifikante Erkenntnisfortschritte worden, und dieses Wissen ist auch von der Wirtschaftspraxis wahrgenommen und absorpiert worden.

\section{Literaturhinweise}

Ajzen, I. (1991): The theory of planned behavior, in: Organizational Behavior and Human Decision Processes, Vol. 50, No. 2, S. 179-211.

Ajzen, I./Fishbein, M. (1977): Attitude-behavior relations: A theoretical analysis and review of empirical research, in: Psychological Bulletin, Vol. 84, No. 5, S. 888-918.

Ajzen, I./Fishbein, M. (2005): The handbook of social psychology, in: Albaracin, D. et al. The Handbook of Attitudes. Mahwah, NJ: Lawrence Erlbaum, S. 173-221. 
Anderson, B.S., Covin, J.G., Slevin, D.P. (2009): Understanding the relationship between entrepreneurial orientation and strategic learning capability: An empirical investigation, in: Strategic Entrepreneurship Journal, Vol. 3, No. 3, S. 218-240.

Anderson, B.S., Kreiser, P.M., Kuratko, D.F., Hornsby, J.S., Eshima, Y. (2015): Reconceptualizing Entrepreneurial Orientation, in: Strategic Management Journal, Vol. 36, S. 1579-1596.

Antoncic, B./Hisrich, R.D. (2001): Intrapreneurship: Construct refinement and cross-cultural validation, in: Journal of Business Venturing, Vol. 16, No. 5, S. 495-527.

Antoncic, B./Hisrich, R.D. (2003): Clarifying the intrapreneurship concept, in: Journal of Small Business and Enterprise Development, Vol. 10, No. 1, S. 7-24.

Arbaugh, J.B., Cox, L.W., Camp, S.M. (2009): Is entrepreneurial orientation a global construct? A multi-country study of entrepreneurial orientation, growth strategy, and performance, in: Journal of Business Inquiry, Vol. 8, No. 1, S. 12-25.

Bacharach, S.B. (1989): Organizational theories: Some criteria for evaluation, in: Academy of Management Review, Vol. 14, No. 4, S. 496-515.

Bagozzi, R.P. (1992): The self-regulation of attitudes, intensions and behavior, in: Social Psychology Quarterly, Vol. 55, S. 178-204.

Bagozzi, R.P./Edwards, J.R. (1998): A general approach for representing constructs in organizational research, in: Organizational Research Methods, Vol. 1, No. 1, S. 45-87.

Becherer, R.C./Maurer, J.G. (1997): The moderating effect of environmental variables on the entrepreneurial and marketing orientation of entrepreneur-led firms, in: Entrepreneurship: Theory \& Practice, Vol. 22, No. 1, S. 47-58.

Brown, T.E., Davidsson, P., Wiklund, J. (2001): An operationalization of Stevenson's conceptualization of entrepreneurship as opportunity-based firm behavior, in: Strategic Management Journal, Vol. 22, No. 10, S. 953-968.

Collier, D./Mahon, J.E. Jr. (1993): Conceptual "stretching" revisited: Adapting categories in comparative analysis, in: The American Political Science Review, Vol. 87, No. 4, S. 845-855.

Coltman, T., Devinney, T.M., Midgley, D.F., Venaik, S. (2008): Formative versus reflective measurement models: Two applications of formative measurement, in: Journal of Business Research, Vol. 61, No. 12, S. 1250-1262.

Covin, J.G./Covin, T.J. (1990): Competitive aggressiveness, environmental context, and small firm performance, in: Entrepreneurship: Theory \& Practice, Vol. 14, No. 4, S. 35-50.

Covin, J.G., Green, K.M., Slevin, D.P. (2006): Strategic process effects on the entrepreneurial orientation-sales growth rate relationship, in: Entrepreneurship: Theory \& Practice, Vol. 30, No. 1, S. 57-81.

Covin, J.G./Lumpkin, G.T. (2011): Entrepreneurial orientation theory and research: Reflections on a needed construct, in: Entrepreneurship: Theory \& Practice, Vol. 35, No. 5, S. 855-872.

Covin, J.G., Prescott, J.E., Slevin, D.P. (1990): The effects of technological sophistication on strategic profiles, structure and firm performance, in: Journal of Management Studies, Vol. 27, No. 5, S. 485-510.

Covin, J.G./Slevin, D.P. (1989): Strategic management of small firms in hostile and benign environments, in: Strategic Management Journal, Vol. 10, No. 1, S. 75-87.

Covin, J.G./Slevin, D.P. (1991): A conceptual model of entrepreneurship as firm behavior, in: Entrepreneurship: Theory \& Practice, Vol. 16, No. 1, S. 7-25. 
Covin, J.G./Slevin, D.P. (2002): The entrepreneurial imperatives of strategic leadership, in: Hitt, M.A. et al. Strategic Entrepreneurship: Creating a New Mindset. Oxford, U.K.: Blackwell Publishers, S. 309-327.

Covin, J.G., Slevin, D.P., Schultz, R.L. (1994): Implementing strategic missions - effective strategic, structural and tactical choices, in: Journal of Management Studies, Vol. 31, No. 4, S. 481-505.

Covin, J.G./Wales, W.J. (2012): The measurement of entrepreneurial orientation, in: Entrepreneurship: Theory \& Practice, Vol. 36, No. 4, S. 677-702.

Davis, J.L. (2007): Firm-level entrepreneurship and performance: An examiniation and extension of relationships and measurements of the entrepreneurial orientation construct, Texas: The University of Texas at Arlington.

Dess, G.G., Lumpkin, G.T., Covin, J.G. (1997): Entrepreneurial strategy making and firm performance: Tests of contingency and configurational models, in: Strategic Management Journal, Vol. 18, No. 9, S. 677-695.

Diamantopoulos, A., Riefler, P., Roth, K.P. (2008): Advancing formative measurement models, in: Journal of Business Research, Vol. 61, No. 12, S. 1203-1218.

Dickson, P.H./Weaver, K.M. (1997): Environmental determinants and individual-level moderators of alliance use, in: Academy of Management Journal, Vol. 40, No. 2, S. 404-425.

Edwards, J.R. (2011): The fallacy of formative measurement, in: Organizational Research Methods, Vol. 14, No. 2, S. 370-388.

Edwards, J.R./Bagozzi, R.P. (2000): On the nature and direction of relationships between constructs and measures, in: Psychological Methods, Vol. 5, No. 2, S. 155-174.

Fazio, R.H. (1990): Multiple porcesses by which attitudes guide behavior: The MODE model as an integrated framework, in: Advances in Experimental Social Psychology, Vol. 23, S. 75-109.

Fazio, R.H., Sanbonmatsu, D.M., Powell, M.C., Kardes, F.R. (1986): On the automatic activation of attitudes, in: Journal of Personality and Social Psychology, Vol. 50, S. 229-238.

Finn, A./Kayande, U. (2004): Scale modification: alternative approaches and their consequences, in: Journal of Retailing, Vol. 80, No. 1, S. 37-52.

Fishbein, M. (1979): A theory of reasoned action: Some applications and implications, in: Howe H.E. Nebraska Symposium on Motivation. Lincoln, NE: University of Nebraska Press, S. 65-116.

George, B.A. (2011): Entrepreneurial orientation: A theoretical and empirical examination of the consequences of differing construct representations, in: Journal of Management Studies, Vol. 48, No. 6, S. 1291-1313.

George, B.A./Marino, L. (2011): The epistemology of entrepreneurial orientation: Conceptual formation, modeling, and operationalization, in: Entrepreneurship: Theory \& Practice, Vol. 35, No. 5, S. 989-1024.

Glasman, L.R./Albarracín, D. (2006): Forming attitudes that predict future behavior: A meta-analysis of the attitude-behavior relation, in: Psychological Bulletin, Vol. 132, No. 5, S. 778-822.

Green, K.M., Covin, J.G., Slevin, D.P. (2008): Exploring the relationship between strategic reactiveness and entrepreneurial orientation: The role of structure-style fit, in: Journal of Business Venturing, Vol. 23, No. 3, S. 356-383.

Hamel, G. (2000): Leading the Revolution, in: Harvard University Press, Cambridge, MA.

Hampden-Turner, C./Trompenaars, F. (2006): Cultural intelligence, in: Group \& Organization Management, Vol. 31, No. 1, S. 56-63. 
Hansen, J.D., Deitz, G.D., Tokman, M., Marino, L.D., Weaver, K.M. (2011): Cross-national invariance of the entrepreneurial orientation scale, in: Journal of Business Venturing, Vol. 26, S. 61-78.

Heavey, C., Simsek, Z., Roche, F., Kelly, A. (2009): Decision comprehensiveness and corporate entrepreneurship: The moderating role of managerial uncertainty preferences and environmental dynamism, in: Journal of Management Studies, Vol. 46, No. 8, S. 1289-1314.

Hirsch, P.M./Levin, D.Z. (1999): Umbrella advocates versus validity police: A life-cycle model, in: Organization Science, Vol. 10, No. 2, S. 199-212.

Hitt, M.A., Boyd, B.K., Li, D. (2004): The state of strategic management research and a vision of the future, in: Ketchen D.J., Bergh D.D. Research Methodology in Strategy and Management. New York, NY: Elsevier, S. 1-31.

Hofstede, G. (1993): Cultural constraints in management theories, in: Academy of Management Executive, Vol. 7, No. 1, S. 81-94.

Hult, G.T.M. (2002): Cultural competitiveness in global sourcing, in: Industrial Marketing Management, Vol. 31, No. 1, S. 25-34.

Hult, G.T.M., Hurley, R.F., Knight, G.A. (2004): Innovativeness: Its antecedents and impact on business performance, in: Industrial Marketing Management, Vol. 33, No. 5, S. 429-438.

Hult, G.T.M./Ketchen, D.J. (2001): Does market orientation matter?: A test of the relationship between positional advantage and performance, in: Strategic Management Journal, Vol. 22, No. 9 , S. 899-906.

Hult, G.T.M., Ketchen, D.J., Arrfelt, M. (2007): Strategic supply chain management: Improving performance through a culture of competitiveness and knowledge development, in: Strategic Management Journal, Vol. 28, No. 10, S. 1035-1052.

Hult, G.T.M., Ketchen, D.J., Nichols, E.L. (2002): An examination of cultural competitiveness and order fulfillment cycle time within supply chains, in: Academy of Management Journal, Vol. 45, No. 3, S. 577-586.

Hult, G.T.M., Snow, C.C., Kandemir, D. (2003): The role of entrepreneurship in building cultural competitiveness in different organizational types, in: Journal of Management, Vol. 29, No. 3, S. 401-426.

Ireland, R.D., Covin, J.G., Kuratko, D.F. (2009): Conceptualizing corporate entrepreneurship strategy, in: Entrepreneurship: Theory \& Practice, Vol. 33, No. 1, S. 19-46.

Ireland, R.D./Webb, J.W. (2007): A multi-theoretic perspective on trust and power in strategic supply chains, in: Journal of Operations Management, Vol. 25, No. 2, S. 482-497.

Jarvis, C.B., MacKenzie, S.B., Podsakoff, P.M. (2003): A critical review of construct indicators and measurement model misspecification in marketing and consumer research, in: Journal of Consumer Research, Vol. 30, No. 2, S. 199-218.

Javalgi, R.G., Todd, P.R., Johnston, W.J., Granot, E. (2012): Entrepreneurship, muddling through, and Indian Internet-enabled SMEs, in: Journal of Business Research, Vol. 65, No. 6, S. 740-744.

Kanter, R.M., North, J., Bernstein, A.P., Williamson, A. (1990): Engines of progress: Designing and running entrepreneurial vehicles in established companies, in: Journal of Business Venturing, Vol. 5, No. 6, S. 415-430.

Kaya, N./Seyrek, I.H. (2005): Performance impacts of strategic orientations: Evidence from turkish manufacturing firms, in: Journal of American Academy of Business, Vol. 6, No. 1, S. 68-71.

Kemelgor, B.H. (2002): A comparative analysis of corporate entrepreneurial orientation between selected firms in the Netherlands and the USA, in: Entrepreneurship \& Regional Development, Vol. 14, No. 1, S. 67-87. 
Kerlinger, F.N./Lee, H.B. (2000): Foundations of Behavioral Research, in: Wadsworth Thompson Learning, Fourth Edition ed, London, U.K.

Khandwalla, P. (1977): The Design of Organizations, Harcourt Brace Jovanovich, New York.

Kirca, A.H., Jayachandran, S., Bearden, W.O. (2005): Market orientation: A meta-analytic review and assessment of its antecedents and impact on performance, in: Journal of Marketing, Vol. 69, No. 2, S. 24-41.

Klein, K.J., Dansereau, F., Hall, R.J. (1994): Levels issues in theory development, data collection, and analysis, in: Academy of Management Review, Vol. 19, No. 2, S. 195-229.

Knight, G.A. (1997): Cross-cultural reliability and validity of a scale to measure firm entrepreneurial orientation, in: Journal of Business Venturing, Vol. 12, No. 3, S. 213.

Kohli, A.K./Jaworski, B.J. (1990): Market Orientation: The construct, research propositions, and managerial implications, in: Journal of Marketing, Vol. 54, No. 2, S. 1-18.

Kollmann, T., Christofor, J., Kuckertz, A. (2007): Explaining individual entrepreneurial orientation: Conceptualisation of a cross-cultural research framework, in: International Journal of Entrepreneurship and Small Business, Vol. 4, No. 3, S. 325-340.

Kothandapani, V. (1971): Validation of feeling, belief and intention to act as three components of attitude and their contribution to prediction of contraceptive behaviour, in: Journal of Personality and Social Psychology, Vol. 19, No. 3, S. 321-333.

Kreiser, P.M., Marino, L.D., Weaver, K.M. (2002): Assessing the psychometric properties of the entrepreneurial orientation scale: A multi-country analysis, in: Entrepreneurship: Theory \& Practice, Vol. 26, No. 4, S. 71-94.

Krueger, N.F., Reilly, M.D., Carsrud, A.L. (2000): Competing models of entrepreneurial intentions, in: Journal of Business Venturing, Vol. 15, No. 5-6, S. 411-432.

Kuratko, D.F./Audretsch, D.B. (2009): Strategic entrepreneurship: Exploring different perspectives of an emerging concept, in: Entrepreneurship: Theory \& Practice, Vol. 33, No. 1, S. 1-17.

Kuratko, D.F., Ireland, R.D., Covin, J.G., Hornsby, J.S. (2005): A model of mid-level managers' entrepreneurial behavior, in: Entrepreneurship: Theory \& Practice, Vol. 29, No. 6, S. 699-716.

Law, K.S./Wong, C.-S. (1999): Multidimensional constructs in structural equation analysis: An illustration using the job perception and job satisfaction constructs, in: Journal of Management, Vol. 25, No. 2, S. 143-160.

Law, K.S., Wong, C.-S., Mobley, W.H. (1998): Toward a taxonomy of multidimensional constructs, in: Academy of Management Review, Vol. 23, No. 4, S. 741-755.

Lumpkin, G.T., Cogliser, C.C., Schneider, D.R. (2009): Understanding and measuring autonomy: An entrepreneurial orientation perspective, in: Entrepreneurship: Theory \& Practice, Vol. 33, No. 1, S. 47-69.

Lumpkin, G.T./Dess, G.G. (1996): Clarifying the entrepreneurial orientation construct and linking it to performance, in: Academy of Management Review, Vol. 21, No. 1, S. 135-172.

Lumpkin, G.T./Dess, G.G. (2001): Linking two dimensions of entrepreneurial orientation to firm performance: the moderating role of environment and industry life cycle, in: Journal of Business Venturing, Vol. 16, No. 5, S. 429-451.

Lyon, D.W., Lumpkin, G.T., Dess, G.G. (2000): Enhancing entrepreneurial orientation research: Operationalizing and measuring a key strategic decision making process, in: Journal of Management, Vol. 26, No. 5, S. 1055-1085.

MacCallum, R.C./Browne, M.W. (1993): The use of causal indicators in covariance structure models: Some practical issues, in: Psychological Bulletin, Vol. 114, No. 3., S. 533-541. 
MacKenzie, S.B., Podsakoff, P.M., Jarvis, C.B. (2005): The problem of measurement model misspecification in behavioral and organizational research and some recommended solutions, in: Journal of Applied Psychology, Vol. 90, No. 4, S. 710-730.

March, J.G./Simon, H.A. (1958): Organizations. Wiley, New York, NY.

Matsuno, K., Mentzer, J.T., Özsomer, A. (2002): The effects of entrepreneurial proclivity and market orientation on business performance, in: Journal of Marketing, Vol. 66, No. 3, S. 18-32.

Merz, G.R./Sauber, M.H. (1995): Profiles of managerial activities in small firms, in: Strategic Management Journal, Vol. 16, No. 7, S. 551-564.

Merz, G.R., Weber, P.B., Laetz, V.B. (1994) : Linking small business management with entrepreneurial growth, in: Journal of Small Business Management, Vol. 32, No. 4, S. 48-60.

Meyer, G.D./Heppard, K.A. (2000): Entrepreneurial strategies: The dominant logic of entrepreneurship, in: Meyer, G.D./Heppard, K.A. Entrepreneurship as Strategy: Competing on the Entrepreneurial Edge. Thousand Oaks, CA: Sage Publications, S. 1-22.

Miles, M.P./Arnold, D.R. (1991): The relationship between marketing orientation and entrepreneurial orientation, in: Entrepreneurship: Theory \& Practice, Vol. 15, No. 4, S. 49-65.

Miller, D. (1983): The correlates of entrepreneurship in three types of firms, in: Management Science, Vol. 29, No. 7, S. 770-791.

Miller, D. (2011), Miller, D. (1983) revisited: A reflection on EO research and some suggestions for the future, in: Entrepreneurship: Theory \& Practice, Vol. 35, No. 5, S. 873-894.

Miller, D./Friesen, P.H. (1978): Archetypes of strategy formulation, in: Management Science, Vol. 24, No. 9, S. 921-933.

Miller, D./Le Breton-Miller, I. (2011): Governance, social identity, and entrepreneurial orientation in closely held public companies, in: Entrepreneurship: Theory \& Practice, Vol. 35, No. 5, S. 1051-1076.

Mintzberg, H. (1973). Strategy making in three modes, in: California Management Review, 16(2), S. 44-53.

Morris, M.H./Paul, G.W. (1987): The relationship between entrepreneurship and marketing in established firms, in: Journal of Business Venturing, Vol. 2, No. 3, S. 247-259.

Naman, J.L./Slevin, D.P. (1993): Entrepreneurship and the concept of fit: A model and empirical tests, in: Strategic Management Journal, Vol. 14, No. 2, S. 137-153.

Noble, C.H., Sinha, R.K., Kumar, A. (2002): Market orientation and alternative strategic orientations: A longitudinal assessment of performance implications, in: Journal of Marketing, Vol. 66, No. 4, S. 25-39.

Nunnally, J.C. (1978). Psychometric Theory, McGraw-Hill, New York, NY.

Obloj, T., Obloj, K., Pratt, M.G. (2010): Dominant logic and entrepreneurial firms' performance in a transition economy, in: Entrepreneurship: Theory \& Practice, Vol. 34, No. 1, S. 151-170.

Olson, J.M./Zanna, M.P. (1993): Attitudes and attitude change, in: Annual Review of Psychology, Vol. 44, S. 117-154.

Petter, S., Straub, D., Rai, A. (2007): Specifying formative constructs in information systems research, in: MIS Quarterly, Vol. 31, No. 4, S. 623-656.

Podsakoff, N.P., Shen, W., Podsakoff, P.M. (2006): The role of formative measurement models in strategic management research: Review, critique, and implications for future research, in: Ketchen, D.J./Bergh, D.D. Research Methodology in Strategy and Management. Oxford, U.K.: Elsevier, S. 197-252. 
Prahalad, C.K./Bettis, R.A. (1986): The dominant logic: A new linkage between diversity and performance, in: Strategic Management Journal, Vol. 7, No. 6, S. 485-501.

Rauch, A., Wiklund, J., Lumpkin, G.T., Frese, M. (2009): Entrepreneurial orientation and business performance: An assessment of past research and suggestions for the future, in: Entrepreneurship: Theory \& Practice, Vol. 33, No. 3, S. 761-787.

Richard, O.C., Barnett, T., Dwyer, S., Chadwick, K. (2004): Cultural diversity in management, firm performance, and the moderating role of entrepreneurial orientation dimensions, in: Academy of Management Journal, Vol. 47, No. 2, S. 255-266.

Rossiter, J.R. (2002): The C-OAR-SE procedure for scale development in marketing, in: International Journal of Research in Marketing, Vol. 19, No. 4, S. 305-335.

Sapienza, H.J./Grimm, C.M. (1997): Founder characteristics, start-up process, and strategy/structure variables as predictors of shortline railroad performance, in: Entrepreneurship: Theory \& Practice, Vol. 22, No. 1, S. 5-24.

Satori, G. (1984): Social Science Concepts: A Aystematic Analysis, in: Sage Publications, Beverly Hills, CA.

Shapero, A. (1982): Social dimensions of entrepreneurship, in: Kent, C. et al. The Encyclopedia of Entrepreneurship. Englewood Cliffs, NJ: Prentice-Hall, S. 72-90.

Shaver, K.G. (1987): Principles of Social Psychology. Winthrop, Cambridge, MA.

Short, J.C., Ketchen, D.J., Shook, C.L., Ireland, R.D. (2010): The concept of "opportunity" in entrepreneurship research: Past accomplishments and future challenges, in: Journal of Management, Vol. 36, No. 1, S. 40-65.

Simsek, Z., Heavey, C., Veiga, J.F. (2010): The impact of CEO core self-evaluation on the firm's entrepreneurial orientation, in: Strategic Management Journal, Vol. 31, No. 1, S. 110-119.

Singh, J. (1995): Measurement issues in cross-national research, in: Journal of International Business Studies, Vol. 26, No. 3, S. 597-619.

Steenkamp, J.-B.E.M./Baumgartner, H. (1998): Assessing measurement invariance in cross-national consumer research, in: Journal of Consumer Research, Vol. 25, No. 1, S. 78-90.

Stetz, P.E., Howell, R., Stewart, A., Blair, J.D., Fottler, M.D. (2000): Multidimensionality of entrepreneurial firm-level processes: Do the dimensions covary?, in: Reynolds P.D. et al.Frontiers of Entrepreneurship Research. Wellesley, MA: Babson College.

Suddaby, R. (2010): Editor's comments: Construct clarity in theories of management and organization, in: Academy of Management ReviewVol. 35, No. 3, S. 346-357.

Tang, J., Tang, Z., Marino, L.D., Zhang, Y., Li, Q. (2008): Exploring an inverted U-shape relationship between entrepreneurial orientation and performance in Chinese ventures, in: Entrepreneurship: Theory \& Practice, Vol. 32, No. 1, S. 219-239.

Venkatraman, N. (1989): The concept of fit in strategy research: Toward verbal and statistical correspondence, in: Academy of Management Review, Vol. 14, No. 3, S. 423-444.

Walter, A., Auer, M., Ritter, T. (2006): The impact of network capabilities and entrepreneurial orientation on university spin-off performance, in: Journal of Business Venturing, Vol. 21, No. 4, S. 541-567.

Wang, C.L. (2008): Entrepreneurial orientation, learning orientation, and firm performance, in: Entrepreneurship: Theory \& Practice, Vol. 32, No. 4, S. 635-657.

Wickliffe, V.P. (2004): Refinement and re-assessment of the consumer decision-making style instrument, in: Journal of Retailing and Consumer Services, Vol. 11, No. 1, S. 9-17. 
Wiklund, J. (1998): Small Firm Growth and Performance: Entrepreneurship and Beyond. Jönköping: Jönköping International Business School.

Wiklund, J. (1999): The sustainability of the entrepreneurial orientation-performance relationship, in: Entrepreneurship: Theory \& Practice, Vol. 24, No. 1, S. 39-50.

Wiklund, J./Shepherd, D.A. (2003): Knowledge-based resources, entrepreneurial orientation, and the performance of small and medium-sized businesses, in: Strategic Management Journal, Vol. 24, No. 13, S. 1307-1314.

Wiklund, J./Shepherd, D.A. (2005): Entrepreneurial orientation and small business performance: A configurational approach, in: Journal of Business Venturing, Vol. 20, No. 1, S. 71-91.

Williams, L.J., Edwards, J.R., Vandenberg, R.J. (2003): Recent advances in causal modeling methods for organizational and management research, in: Journal of Management, Vol. 29, No. 6, S. 903-936.

Zahra, S.A. (1993): A conceptual model of entrepreneurship as firm behavior: A critique and extension, in: Entrepreneurship: Theory \& Practice, Vol. 17, No. 4, S. 5-21.

Zahra, S.A./Covin, J.G. (1995): Contextual influences on the corporate entrepreneurship-performance relationship: A longitudinal analysis, in: Journal of Business Venturing, Vol. 10, No. 1, S. 43-58.

Zahra, S.A., Jennings, D.F., Kuratko, D.F. (1999): The antecedents and consequences of firm-level entrepreneurship: The state of the field, in: Entrepreneurship: Theory \& Practice, Vol. 24, No. 2, S. 47-67.

Zahra, S.A./Neubaum, D.O. (1998): Environmental adversity and the entrepreneurial activities of new ventures, in: Journal of Developmental Entrepreneurship, Vol. 3, No. 2, S. 123-140.

Simone A. Schweiger, Dr., Dozentin und Habilitandin an der Universität Bern, Abteilung für Unternehmensführung und Entrepreneurship

Anschrift: Universität Bern, Engehaldenstrass 4, CH-3012 Bern, e-Mail: simone.schweiger@imu.unibe.ch

Artur Baldauf, Prof. Dr., Direktor der Abteilung für Unternehmensführung und Entrepreneurship, Universität Bern

Anschrift: Universität Bern, Engehaldenstrasse 4, CH-3012 Bern, e-Mail: baldauf@imu.unibe.ch 